\title{
A frequent variant in the Japanese population determines quasi-Mendelian inheritance of rare retinal ciliopathy
}

Konstantinos Nikopoulos 1,2,20, Katarina Cisarova1,20, Mathieu Quinodoz'1,20, Hanna Koskiniemi-Kuendig', Noriko Miyake ${ }^{3}$, Pietro Farinelli', Atta Ur Rehman', Muhammad Imran Khan 4,5, Andrea Prunotto (1) 1, Masato Akiyama ${ }^{6}$, Yoichiro Kamatani (1) 6, Chikashi Terao (1) 6, Fuyuki Miya7, Yasuhiro Ikeda ${ }^{8}$, Shinji Ueno9, Nobuo Fuse ${ }^{10}$, Akira Murakami (iD ${ }^{11}$, Yuko Wada ${ }^{12}$, Hiroko Terasaki ${ }^{9}$, Koh-Hei Sonoda ${ }^{8}$, Tatsuro Ishibashi ${ }^{8}$, Michiaki Kubo ${ }^{13}$, Frans P.M. Cremers ${ }^{4,5}$, Zoltán Kutalik (10 ${ }^{14}$, Naomichi Matsumoto (1) ${ }^{3}$, Koji M. Nishiguchi ${ }^{15,16}$, Toru Nakazawa ${ }^{15,16}$ \& Carlo Rivolta (1) 1,17,18,19

Hereditary retinal degenerations (HRDs) are Mendelian diseases characterized by progressive blindness and caused by ultra-rare mutations. In a genomic screen of 331 unrelated Japanese patients, we identify a disruptive Alu insertion and a nonsense variant (p.Arg1933*) in the ciliary gene $R P 1$, neither of which are rare alleles in Japan. p.Arg1933* is almost polymorphic (frequency $=0.6 \%$, amongst 12,000 individuals), does not cause disease in homozygosis or heterozygosis, and yet is significantly enriched in HRD patients (frequency = $2.1 \%$, i.e., a 3.5 -fold enrichment; $p$-value $=9.2 \times 10^{-5}$ ). Familial co-segregation and association analyses show that p.Arg1933* can act as a Mendelian mutation in trans with the Alu insertion, but might also associate with disease in combination with two alleles in the EYS gene in a non-Mendelian pattern of heredity. Our results suggest that rare conditions such as HRDs can be paradoxically determined by relatively common variants, following a quasiMendelian model linking monogenic and complex inheritance.

\footnotetext{
${ }^{1}$ Unit of Medical Genetics, Department of Computational Biology, University of Lausanne, 1015 Lausanne, Switzerland. ${ }^{2}$ Service of Medical Genetics, Lausanne University Hospital (CHUV), 1011 Lausanne, Switzerland. ${ }^{3}$ Department of Human Genetics, Yokohama City University Graduate School of Medicine, Yokohama 2360004, Japan. ${ }^{4}$ Department of Human Genetics, Radboud University Medical Center, 6500 HB Nijmegen, The Netherlands. ${ }^{5}$ Donders Institute for Brain, Cognition and Behaviour, Radboud University Nijmegen, 6525 GA Nijmegen, The Netherlands. ${ }^{6}$ Laboratory for Statistical Analysis, RIKEN Center for Integrative Medical Sciences, Yokohama 230-0045, Japan. ${ }^{7}$ Department of Medical Science Mathematics, Medical Research Institute, Tokyo Medical and Dental University, Tokyo 1138510, Japan. ${ }^{8}$ Department of Ophthalmology, Graduate School of Medical Sciences, Kyushu University, Fukuoka 812-8582, Japan. ${ }^{9}$ Department of Ophthalmology, Nagoya University Graduate School of Medicine, Nagoya 466-8550, Japan. ${ }^{10}$ Department of Integrative Genomics, Tohoku Medical Megabank Organization, Sendai 980-8573, Japan. ${ }^{11}$ Department of Ophthalmology, Juntendo University School of Medicine, Tokyo 113-8421, Japan. ${ }^{12}$ Yuko Wada Eye Clinic, Sendai $980-0011$, Japan. ${ }^{13}$ RIKEN Center for Integrative Medical Sciences, Yokohama 230-0045, Japan. ${ }^{14}$ Institute of Social and Preventive Medicine, Lausanne University Hospital, 1011 Lausanne, Switzerland. ${ }^{15}$ Department of Ophthalmology, Tohoku University Graduate School of Medicine, Sendai 980-8574, Japan. ${ }^{16}$ Department of Advanced Ophthalmic Medicine, Tohoku University Graduate School of Medicine, Sendai 980-8574, Japan. ${ }^{17}$ Department of Genetics and Genome Biology, University of Leicester, Leicester LE1 7RH, UK. ${ }^{18}$ Institute of Molecular and Clinical Ophthalmology Basel (IOB), 4031 Basel, Switzerland. ${ }^{19}$ University of Basel, 4001 Basel, Switzerland. ${ }^{21}$ These authors contributed equally: Konstantinos Nikopoulos, Katarina Cisarova, Mathieu Quinodoz. Correspondence and requests for materials should be addressed to C.R. (email: carlo.rivolta@iob.ch)
} 
$\mathrm{T}$ ogether with intellectual disabilities, hereditary retinal degenerations (HRDs, comprising retinitis pigmentosa and allied diseases) represent a group of conditions for which both genetic and allelic heterogeneity is the highest in humans $\mathrm{s}^{1,2}$. To date, almost 300 genes and thousands of mutations have been identified as causative of HRD, and the detection of novel disease genes and variants continues at a steady pace (RetNet database: https://sph.uth.edu/retnet/). Considering that the overall prevalence of HRDs does not exceed 1 in 2000 individuals, the average contribution of any given HRD gene to the disease is incredibly small. Similarly, apart from two DNA variants that appear to be relatively frequent in the general population and determine a specific form of the disease (p.Asn1868Ile and p.Gly863Ala in $A B C A 4)^{3,4}$, most mutations are so rare that they are seldom detected in more than one pedigree, worldwide. In addition, although HRDs affect people from the five continents, their specific allelic assortment seems to be population-specific ${ }^{5,6}$. For instance, similar to other islanders or groups of people that have experienced relative historical isolation, Japanese carry certain alleles, including pathogenic ones, which are not found elsewhere in the world 7 . Furthermore, lack of significant reduction in fitness before the reproductive age, associated with such an elevated heterogeneity, have led to the consequence that the number of recessive mutations that are detected heterozygously in the general, unaffected population is remarkably high and may affect up to one person in two ${ }^{8}$.

Despite this extraordinary variability and abundance of mutations, HRDs are almost invariantly inherited as a monogenic, Mendelian trait, for which the presence of only one (dominant) or two (recessive) mutations in the same gene, genome-wide, is at the same time a necessary and sufficient condition for pathogenicity ${ }^{9}$. At the other end of the spectrum of ocular conditions having a genetic component lies age-related macular degeneration (AMD), another retinal disease affecting people aged 50 and over. AMD is a bona fide complex disease with a relatively high prevalence ( 1 in 13 individuals), favored by the presence of polymorphic SNPs, highly penetrant rare variants, and environmental factors ${ }^{10}$. Between these two pillars of inheritance, there is an intermediate zone, consisting in a few examples for which extremely rare mutations in more than one gene are associated with Bardet-Biedl syndrome, a retinal ciliopathy displaying sometimes digenic triallelic inheritance ${ }^{11-13}$.

$R P 1$ is one of the several HRD genes identified to date, and one of the few causing disease by more than one Mendelian pattern of inheritance. Originally described as linked to autosomal dominant retinitis pigmentosa (adRP) ${ }^{14-16}$, a subtype of HRD, it was later shown to be associated with a recessive form of the same disease $(\operatorname{arRP})^{17}$. To date, at least 60 mutations have been reported in $R P 1$, most of which cluster within its last exon (exon 4 ), cumulatively accounting approximately for $5.5 \%$ and up to $4.5 \%$ of all adRP and arRP cases, respectively ${ }^{18,19}$. However, some DNA variants at the far $3^{\prime}$ end of the gene, including nonsense variants, appear not to cause disease, at least not according to a dominant or recessive pattern of inheritance ${ }^{20,21} . R P 1$ encodes a multi-modular protein of 2156 amino acids, which is a member of the doublecortin family and is present in the ciliary axoneme of both rods and cones, the light-sensing neurons of the retina ${ }^{22,23}$. Mutations in RP1 thus determine visual loss as a consequence of a ciliopathic phenotype affecting these specialized cell types.

Following the screen of a large set of Japanese HRD patients, we identify three mutations in the $R P 1$ gene: a mobile Alu element insertion in exon 4, a novel frameshift mutation, and a nonsense variant in the far 3 ' part of the coding sequence. While the first two variants behave as classical recessive Mendelian alleles, NM_006269.1:c.5797C >T/p.Arg1933* appears to cause disease according to a more complex pattern of inheritance.
When present in trans with respect to the Alu insertion, it acts as a Mendelian mutation. Furthermore, despite being enriched in patients vs. controls, p.Arg1933* is completely benign in homozygosis or in heterozygosis. By performing an association test between $28 \mathrm{HRD}$ patients, heterozygous carriers of this nonsense allele, and 3554 controls, we find that p.Arg1933* may be pathogenic not only as a Mendelian allele, but also in association with variants elsewhere in the genome, and in particular with two DNA changes in another ciliary gene, EYS.

\section{Results}

An $A l u$ insertion in RP1 is a prevalent cause of HRD in Japan. In the framework of a screening effort of 331 unrelated Japanese patients, we identified a novel, unusual mutation by wholegenome sequencing, consisting in the insertion of a mobile Alu element in exon 4 of the RP1 gene (m1, or NM_006269.1: c.4052_4053ins328/p.Tyr1352Alafs ${ }^{*}$ ) in a female individual from a recessive HRD family. This insertion caused the disruption of the reading frame by introducing 328 additional nucleotides, including a premature termination codon in the canonical RP1 coding sequence. The mother of the proband was heterozygous for this variant and the proband's affected brother was also a homozygote, in support of the notion that this was indeed a recessive HRD mutation (Fig. 1a). Targeted screening for this Alu insertion in the remaining 330 patients (all forms of HRDs, isolate or recessive cases, not genetically pre-screened), as well as in 524 Japanese controls, available for direct testing of $\mathrm{m} 1$, identified 15 other affected and unrelated individuals and one heterozygous control carrying this insertion. In total, six patients were homozygous for the mutation (12 alleles), which co-segregated with the disease as a classical Mendelian, recessive allele, whenever this could be tested, while 10 carried it heterozygously. Altogether, these findings indicate that this Alu insertion is not only clearly pathogenic, but it is also a rather prevalent cause of retinal degeneration within the Japanese islands at the level of a single allele (1.8\% of all HRD Japanese patients), possibly second only to the most frequent mutation so far identified in this country, i.e., NM_001142800.1:c.4957dup in EYS ${ }^{24-26}$.
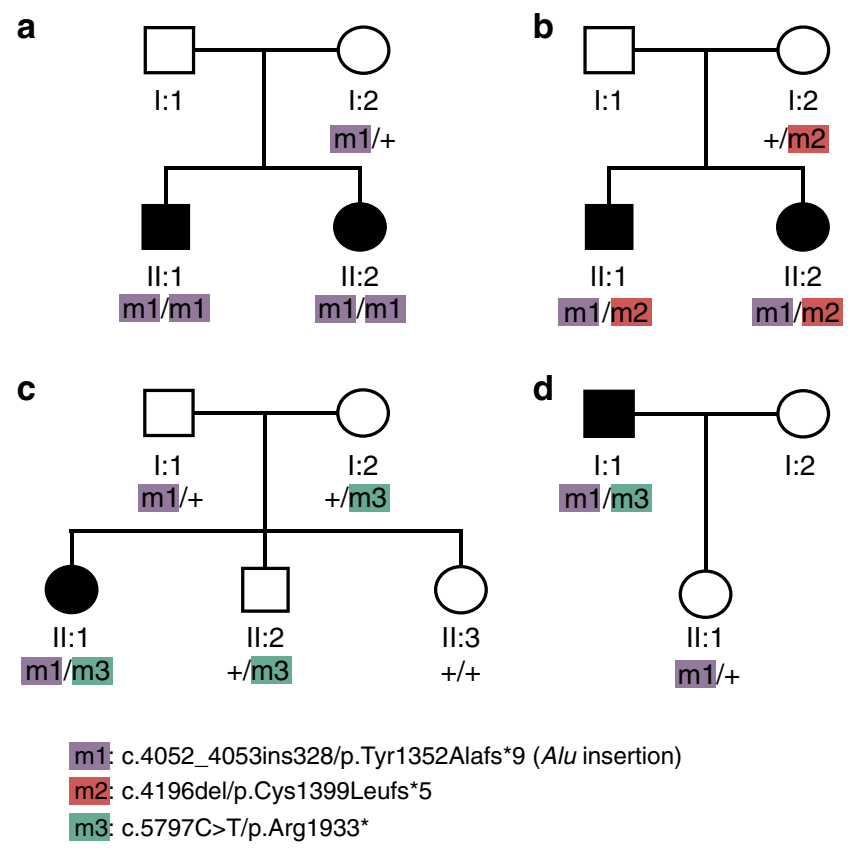

Fig. 1 Segregation analysis of the RP1 mutations found in this study. Pedigrees of representative families are shown 
Remarkably, 6 of the 10 individuals who carried the Alu insertion heterozygously were in fact compound heterozygotes for either of two other changes in RP1: a novel frameshift mutation (c.4196del/p.Cys1399Leufs ${ }^{\star} 5, \mathrm{~m} 2$, two unrelated individuals) and a nonsense variant c.5797 C > T/p.Arg1933* $(\mathrm{m} 3$, four unrelated individuals) that was previously identified in the general population and is present in dbSNP as entry \#rs118031911 (Supplementary Table 1). Again, both variants, detected by direct Sanger sequencing, co-segregated with the disease in relevant families, according to an autosomal recessive pattern of inheritance (Fig. 1b-d).

m3 is enriched in patients, but does cause HRD per se. Frameshift c.4196del/p.Cys1399Leufs ${ }^{\star}$ (m2) was absent from 3480 Japanese control chromosomes and was reported in the gnomAD database $^{27}$ to have an allele frequency of $5.44 \times 10^{-5}$ in East Asia, indicating that this DNA variant is a very rare allele, as it is the case for most HRD mutations.

In contrast, the rs118031911/T allele (m3), despite being virtually absent in many world populations, was found to be relatively frequent in East Asians (Supplementary Fig. 1), and probably too frequent to be a Mendelian allele for HRD, according to the Hardy-Weinberg model. In particular, our direct screening of 12,379 Japanese individuals with no retinal degeneration showed the presence of rs118031911/T in 145 subjects, 142 heterozygotes and 3 homozygotes (148 alleles), validating the notion that this DNA variant is in fact almost polymorphic in Japan (allele frequency $=0.6 \%$ ). All these subjects were examined by fundoscopy and, in addition, we evaluated clinically one of the three homozygotes (the only one who could be re-assessed, in agreement with our Institutional Review Board protocol) by a very thorough ophthalmological examination. At age 28 years old, she had no visual symptoms and displayed no ocular abnormalities: she had normal visual acuity (20/20 in both eyes), intact visual field (Goldmann perimetry), and no evidence of retinal degeneration through slit lamp examination and fundoscopy. Furthermore, optical coherence tomography imaging, used to assess detailed retinal structures, showed no signs of retinal thinning and electroretinogram, a test allowing objective detection of minimal retinal dysfunction even in the absence of subjective symptoms, showed normal responses. Finally, absence of lateonset HRD, who could have escaped detection in a 28 -year-old individual, was confirmed by the assessment of the fundi of the other two rs118031911/T homozygotes, who displayed no signs of retinal degeneration at ages of 78 and 79 years, respectively. Overall, both population based-data and direct clinical assessments confirm that rs118031911/T does not cause HRD per se, in heterozygosis or in homozygosis.

However, specific screening for the rs118031911/T allele in the same cohort of 331 Japanese HRD patients mentioned above led to the identification of 10 additional heterozygotes (14 alleles in total) showing that its frequency in HRD patients was $2.1 \%$ (14 alleles out of 662) (Supplementary Fig. 1). The 3.5 -fold enrichment of rs $118031911 / \mathrm{T}$ in patients vs. controls (148 alleles out of $24,758=0.6 \%)$ was highly significant [ $p$ value $=9.2 \times 10^{-5}$, threshold $=0.05 / N, N=1$, by Fisher's exact test $(24,610: 148$ vs. $648: 14)$ ], indicating that this relatively common variant has in fact an effect on retinal health. WES analysis of these 10 patients detected no mutations in HRD genes that could explain their phenotype, according to a Mendelian fashion of inheritance. Considering that rs118031911/T introduces a nonsense codon in the $R P 1$ open reading frame and was found in trans with respect to the Alu insertion in some patients, it is not unlikely that it could represent a hypomorphic variant contributing to the mutational load of genes involved in retinal homeostasis. In other words, despite being benign when considered as a Mendelian allele (monoallelically or biallelically), rs118031911/T could exert a pathogenic function in conjunction with DNA changes in other known HRD genes, according to an oligogenic pattern of inheritance that was previously modeled for hereditary ciliopathies $^{28-30}$.

A non-Mendelian pattern of inheritance for $\mathbf{m} 3$. We tested this hypothesis by assessing enrichment of nonsynonymous, rare, and low-frequency variants (minor allele frequency between $0.1 \%$ and $5 \%$, according to published literature; further details in Methods) in $\mathrm{m} 3$ carriers. We analyzed the 10 patients mentioned above, as well as 18 additional patients with the same genotype (heterozygous for rs118031911/T, with no other recognized mutations in RP1 and no mutations in HRD genes that could explain their phenotype), identified following a targeted screening of 713 Japanese HRD cases from another internal cohort (Supplementary Table 1). Specifically, we performed an association test between these 28 individuals and 3554 Japanese controls from the 3.5KJPN database ${ }^{31}$ by considering all 228 bona fide HRD genes $^{32}$ from the RetNet database (Supplementary Table 2) that could produce multiallelic inheritance of HRD in $\mathrm{m} 3$ heterozygotes, in line with previous protocols involving similar analyses ${ }^{33-38}$. Cryptic relatedness among patients, as well as the presence of additional, undetected $R P 1$ mutations in trans with respect to rs118031911/T were excluded prior to performing the test (Supplementary Table 3 and Supplementary Data 1). The association analysis identified two variants that were significantly enriched in patients vs. controls (Table 1, Fig. 2). Interestingly, although they were not in linkage disequilibrium, both variants belonged to the gene EYS (NM_001142800.1:c.2528 G > A;p.Gly843Glu and c.8805 C > A;p.Tyr2935*, p-values = $5.6 \times 10^{-5}$ and $1.9 \times 10^{-4}$, respectively; threshold $=2.8 \times 10^{-4}=$ $0.05 / N$, where $N=178$, by Fisher's exact test), possibly highlighting a mechanism of pathogenesis directly involving the proteins EYS and RP1. Indeed, a third DNA change within EYS (c.4957del;p.Ser1653Valfs ${ }^{\star} 26$ ) ranked 3rd in the list of associated variants, even if its $p$-value did not reach statistical significance after Bonferroni correction. Furthermore, we performed the same analysis by considering not only variants from RetNet sequences, but from the whole human exome, in both cohorts. In support of the data obtained, the two significant hits detected in the HRD gene set were also the two top hits detected exome-wide, even though no variant reached the threshold for exome-wide significance. Altogether, these results indicate that the rs118031911/ T nonsense could act in concert with at least two DNA changes (and possibly with more) to determine a pathological phenotype in a non-Mendelian fashion.

Based on previous data on Bardet-Biedl syndrome ${ }^{11}$, we tested a digenic diallelic vs. triallelic mode of action for rs118031911/T on HRD, by comparing the frequency of this variant in patients for whom the molecular causes of retinal degenerations were identified (i.e., solved cases) vs. unsolved HRD cases vs. controls. As expected, a comparison of unsolved vs. controls showed significance, as reported above, whereas solved vs. controls did not show any significant enrichment for rs118031911/T (6 rs118031911/T variants over 722 alleles for solved vs. 148 over 24,758 for controls, $p$-value $=0.46, \mathrm{OR}=$ $1.4, \mathrm{CI}=0.50-3.14$, by Fisher's exact test). Comparison of solved vs. unsolved HRD cases showed borderline nonsignificant enrichment for rs118031911/T in unsolved cases $(p$-value $=0.07, \mathrm{OR}=2.30, \mathrm{CI}=0.94-6.76$, by Fisher's exact test), possibly indicating that either well-defined triallelism does 
Table 1 Summary of the results of the association study

\begin{tabular}{|c|c|c|c|c|c|c|}
\hline Gene & Variant & Frequency in cases & Frequency in controls & OR & $95 \% \mathrm{Cl}(\mathrm{OR})$ & $p$-value \\
\hline$\overline{E Y S}$ & NM_001142800.1:c.2528G>A; p.Gly843Glu & 0.125 & 0.017 & 8.23 & $3.08-18.78$ & $5.6 \mathrm{E}-05^{\star}$ \\
\hline EYS & NM_001142800.1:c.8805C>A; p.Tyr2935* & 0.054 & 0.002 & 33.30 & $5.86-128.76$ & $1.9 \mathrm{E}-04^{\star}$ \\
\hline EYS & NM_001142800.1:c.4957del; p.Ser1653Valfs`26 & 0.054 & 0.004 & 13.85 & $2.62-46.83$ & $1.9 \mathrm{E}-03$ \\
\hline USH2A & NM_206933.2:c.2802T>G; p.Cys934Trp & 0.036 & 0.003 & 14.55 & $1.60-63.22$ & 0.010 \\
\hline PDZD7 & NM_024895.4:c.1267G>A; p.Ala423Thr & 0.036 & 0.003 & 13.38 & $1.48-57.70$ & 0.012 \\
\hline EYS & NM_001142800.1:c.7394C>G; p.Thr2465Ser & 0.089 & 0.029 & 3.28 & $1.01-8.30$ & 0.024 \\
\hline$B B I P 1$ & NM_001243783.2:c.112T>C; p.Ser38Pro & 0.036 & 0.005 & 7.70 & $0.87-31.35$ & 0.032 \\
\hline
\end{tabular}

Note: The top 10 hits from this test are shown

$O R$ odds ratio, $\mathrm{Cl}$ confidence interval

${ }^{*} p$-values retaining statistical significance

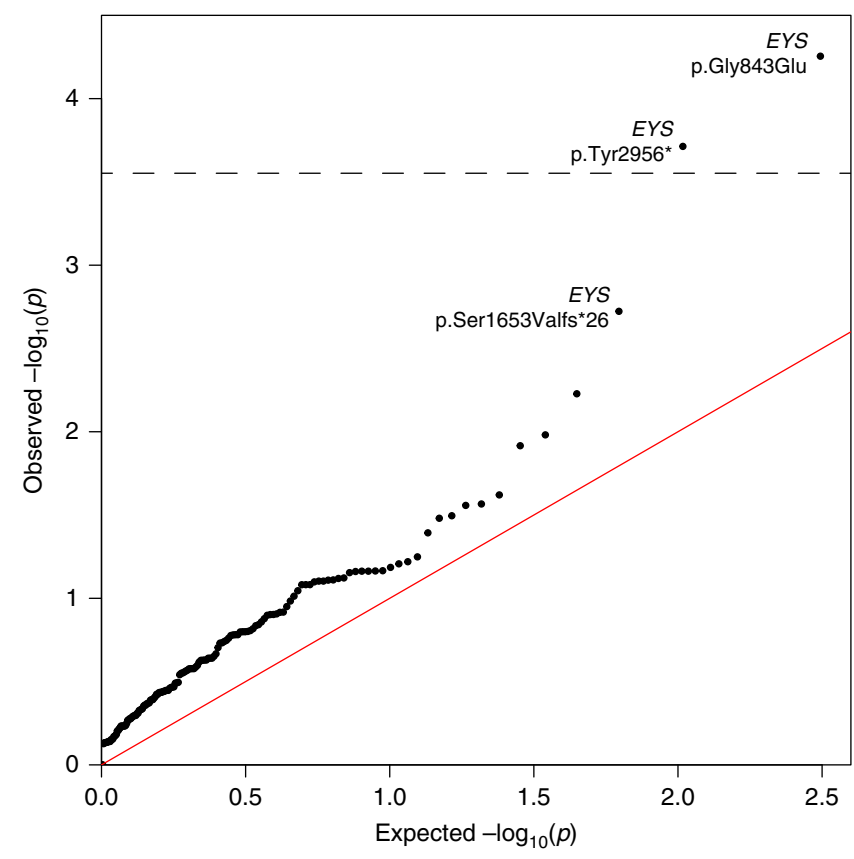

Fig. 2 Results of the association study. Quantile-quantile (Q-Q) plot of rare/low-frequency non-synonymous variants in HRD genes in 28 patients heterozygous for rs118031911/T vs. 3554 Japanese controls. The significance threshold is indicated by the dotted line

not take place for this variant or that we did not have enough power to detect it.

\section{Discussion}

The extreme genetic heterogeneity of retinal degenerations, together with the elevated number of pathogenic and hypomorphic changes in HRD genes that are detected in the unaffected population, have evoked the theoretical possibility that non-Mendelian, oligogenic inheritance could be responsible for these conditions ${ }^{9}$. Digenic heredity has been clearly demonstrated for specific combinations of mutations $s^{39-41}$ in particular pedigrees or in individual cases, including digenic triallelic transmission of Bardet-Biedl syndrome ${ }^{11,42}$. For these patients, the presence of two (diallelic) or three (triallelic) mutations at two different loci (digenism) causes disease, presumably by compromising the overall function of gene products that belong to the same complex or are part of the same biochemical pathway. This model seems to be particularly true for genes encoding for proteins that form or play a role within the cell primary cilium, according to the paradigm of mutational load put forward by $\mathrm{N}$. Katsanis and coworkers ${ }^{43}$. In these instances, accumulation of rare variants (which individually may have a little effect) in multiple ciliary genes can produce a pathological phenotype that is connected to ciliary function and result in a ciliopathy, including retinal ciliopathies ${ }^{44-46}$.

Intriguingly, despite our association test was not limited to ciliary genes, both our significant hits lie within a ciliary gene, EYS. In primates, the EYS protein has been shown to physically co-localize with RP1 in the ciliary axoneme of photoreceptors and is thought to play a role in the structural organization and maintenance of these cells' apical part, the outer segment $(\mathrm{OS})^{47}$. This functional role is further supported by studies in zebrafish, where EYS knockouts show progressive retinal degeneration due to mis-localization of specific OS proteins and the disruption of F-actin filaments ${ }^{48,49}$, a key component not only for the integrity, but also for the morphogenesis of the $\mathrm{OS}^{50}$. In a similar fashion, targeted disruption of the $R P 1$ gene in mice leads to defects of the OS, because of the incorrect stacking of its discs ${ }^{51}$. The co-localization of RP1 and EYS, as well as their common role in the homeostasis of the OS, strongly indicates that they may have synergic functions and that pathogenesis could occur in a digenic fashion.

In this work we show that two specific $R P 1$ alleles are responsible for a relatively large number of Mendelian HRD cases in Japan. Interestingly, none of these two changes is a rare allele at all, compared to the average frequencies of classical $\mathrm{HRD}$ mutations. The first, the c.4052_4053ins328/p.Tyr1352Alafs ${ }^{*}$ Alu element insertion in $R P 1(\mathrm{ml})$, seems to be the second most common HRD recessive mutation described so far in Japan, and its frequency may even be underestimated, since insertional events of mobile elements are difficult to detect by conventional screening techniques. The second variant, c.5797 C > T/p.Arg1933* or rs118031911/T (m3), is even more frequent, and by far more interesting. Despite introducing a premature stop codon in the $R P 1$ open reading frame, this DNA change is almost polymorphic in East Asia and does not cause disease either in heterozygous or homozygous carriers. However, this same change may act as pathogenic allele in a Mendelian fashion (with another RP1 mutation in trans), or in association with rare variants in at least another gene, according to a non-Mendelian, possibly oligogenic pattern of inheritance. Although we currently ignore the molecular mechanisms leading to this unusual model of pathogenicity, it is probably the consequence of an increased global mutational load with threshold effect, determined by the accumulation of variants with different pathogenic potential. The presence of one or of two rs118031911/T alleles likely produces a load that is below this pathological threshold, while the co- 


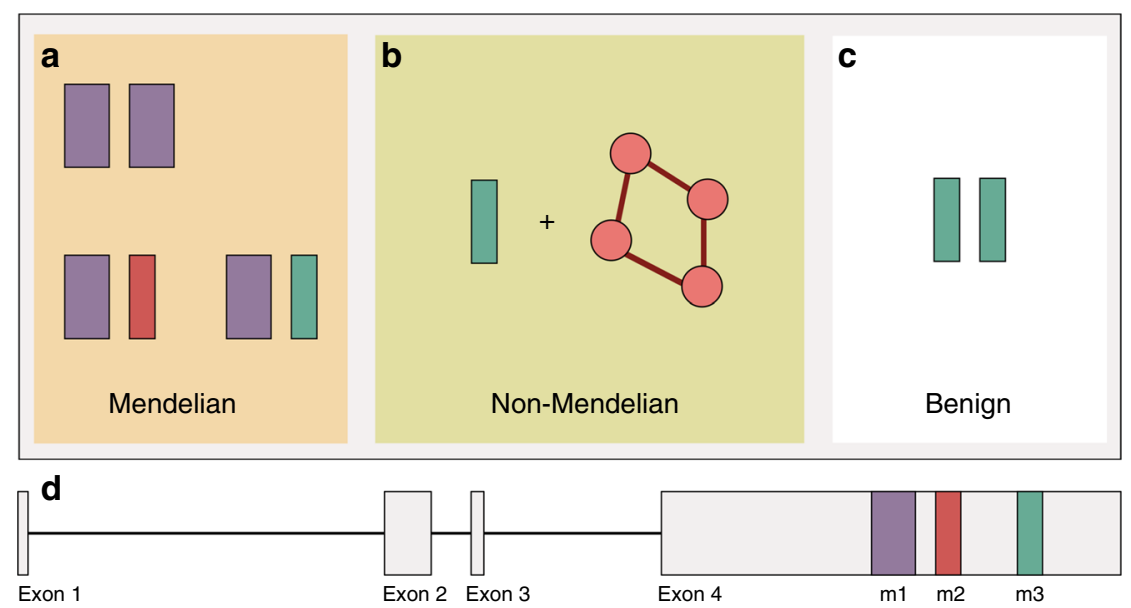

Fig. 3 Schematic representation of the inheritance pattern of the identified mutations in RP1, highlighting the concept of rs118031911/T-mediated quasiMendelian inheritance of HRDs. a In trans with respect to the Alu element insertion (m1, or c.4052_4053ins328/p.Tyr1352Alafs*9), m3 (rs118031911/T, or c.5797C $>$ T/p.Arg1933*) results in autosomal recessive inheritance of the disease, similar to $\mathrm{m} 1 \mathrm{in}$ a homozygous state or in a compound heterozygous combination with $\mathrm{m} 2$ (c.4196del/p.Cys1399Leufs ${ }^{\star}$ ). b Combinations of the hypomorphic $\mathrm{m} 3$ allele with additional hypomorphs and/or heterozygous recessive alleles in other genes result in disease following a non-Mendelian pattern, whereas (c) homozygosis for m3 has no pathological consequences. d Structure of RP1: exons are represented by boxes, connected by solid lines (introns). The relative positions of $\mathrm{m} 1$, $\mathrm{m} 2$, and $\mathrm{m} 3$ are also indicated

occurrence of extra variants could result in the crossing of such a limit for normal retinal homeostasis. This hypothesis is supported by the evidence that $\mathrm{rs} 118031911 / \mathrm{T}$ is pathogenic in conjunction with a very severe mutation, i.e., the insertion of an Alu element in $R P 1$ 's exon 4 mentioned above, which completely ablates the open reading frame of the gene. We term this model of inheritance quasiMendelian, to define the differential behavior (Mendelian or nonMendelian) that specific alleles may have with respect to different genotypes at the same locus or elsewhere in the genome.

In conclusion, it seems that, at least for RP1-associated HRD, disorders displaying a Mendelian pattern of inheritance may also genetically behave like multigenic conditions, for which both polymorphic (having a low effect) and rare (having a rather high effect) variants can determine pathogenesis (Fig. 3). The findings from our study require further replication, ideally in other East Asian cohorts. However, the low prevalence of HRD and the even lower percentage of HRD patients carrying rs118031911/T limit our ability to assemble cohorts of sufficient power at this time, which would not only enable us to validate our findings but also to propose more defined models of pathogenicity. To better illustrate this: the identification of the 28 heterozygotes reported in this study corresponds to the screening of roughly 5-10 million Japanese individuals. Nevertheless, our work provides a proof of concept that a non-negligible proportion of HRDs can be caused by inheritance mechanisms that transcend the Mendelian model, to be investigated in detail by future, very large-scale and population-specific sequencing endeavors, such as for instance the 100,000 genomes project ${ }^{52}$. Furthermore, our findings suggest that oligogenic heredity of human diseases (and perhaps of other traits) may not be limited to a low number of cases with ultra-rare conditions, as shown up to now ${ }^{34,35,53,54}$, but could extend to more frequent phenotypes and represent a bridge between monogenic and complex inheritance.

\section{Methods}

Subjects. The study was initiated following the approval by the Institutional Review Boards of our respective Institutions (University of Lausanne, Yokohama City University Graduate School of Medicine, Radboud University Medical Center, Kyushu University, Nagoya University Graduate School of Medicine, Tohoku Medical Megabank Organization, Juntendo University School of Medicine, Tohoku University Graduate School of Medicine, and Tokyo Medical and Dental University). All subjects provided written informed consent, and the study was conducted in adherence with the Declaration of Helsinki.
Tohoku University School of Medicine, Kyushu University School of Medicine, and Nagoya University School of Medicine, all based in Japan, were the centers where all Japanese patients with HRD were recruited. HRD was diagnosed clinically after excluding possible secondary causes of retinal degeneration such as toxicity and uveitis. Final diagnosis required the presence of reduced electroretinogram (ERG) responses, visual field loss, and funduscopic abnormalities consistent with retinal degeneration (retinal vascular narrowing and abnormalities of the retinal pigment epithelium etc.) symmetrically in both eyes.

Genotypes from individuals without HRD were collected from both published and unpublished databases [the BioBank Japan Project $(N=12,379)$, the ToMMo Japanese Reference Panel Project (3.5KJPN release, $N=3554)^{31}$, the Tohoku University School of Medicine $(N=95)$, the Yokohama City University Graduate School of Medicine $(N=429)$ ] or were obtained experimentally by direct genotyping of genomic DNA, with standard molecular biology techniques.

Summary phenotypes of carriers of $\mathrm{m} 1, \mathrm{~m} 2$, or $\mathrm{m} 3$ mutations are listed in Supplementary Table 1.

Whole genome sequencing and analysis. Genome sequencing of the first index patient was performed using the sequencing platform by Complete Genomics ${ }^{55}$. Sequence reads were mapped to the human reference genome (NCBI build 37) and variants were called genome-wide. These included: single-nucleotide variants (SNVs), copy-number variations (CNVs), as well as structural variations (SVs) such as Alu element insertions and/or chromosomal rearrangements. Data were extracted from MasterVar files and other relevant matrices by ad hoc Perl, bash, and $\mathrm{R}$ scripts, available upon request. Assessment of pathogenic variants was performed as previously described ${ }^{56}$

Screening for the Alu element insertion. In order to screen for the presence of the Alu element in exon 4 of RP1 distinct pair of primers were designed (forward: 5'-AGGCTTGTTTCCTAGGAGAGGT-3', reverse: 5' -

TTCTGCTTCTTTTTCACTTAGGC-3') using the CLCbio Genomics Workbench (Qiagen, Hilden, Germany).

PCR amplification was performed in a $20 \mu \mathrm{l}$ total volume containing $20 \mathrm{ng}$ genomic DNA, $1 \times$ GoTaq buffer, $0.5 \mathrm{mM}$ dNTPs, $10 \mu \mathrm{M}$ of each primer, and 2 units $(5 \mathrm{U} / \mu \mathrm{l})$ of GoTaq polymerase (Promega, Madison, Wisconsin). PCR products were separated following agarose gel electrophoresis. PCR products displaying abnormal size profiles were purified (ExoSAP-IT, USB, Cleveland Ohio) and a sequencing reaction was performed in a total volume of $5 \mu \mathrm{l}$ using $1 \mu \mathrm{l}$ primer $3.3 \mu \mathrm{M}, 0.5 \mu \mathrm{l}$ BigDye Terminator v1.1, and $1 \mu \mathrm{l}$ of the provided Buffer (Applied Biosystems, Foster City, California) Big Dye terminator cycle sequencing kit on an ABI 3130xl Genetic Analyzer (Applied Biosystems).

For this screening, we used 524 controls from the Tohoku University School of Medicine $(N=95)$ and the Yokohama City University Graduate School of Medicine $(N=429)$.

Whole exome sequencing and analysis. Paired-end DNA sequencing libraries of 28 individuals were generated using Aglilent SureSelect Human All ExonV6 kit (Agilent Technologies, CA, USA) by Novogene Co., Ltd., Hong Kong. One microgram of genomic DNA per sample was fragmented into 180-280 bp fragments by hydrodynamic shearing (Covaris, Massachusetts, USA). After the 
reparation of the $3^{\prime}$ and 5 ' ends and the adenylation of the 3 ' ends, paired-end adaptors were ligated to the DNA fragments. DNA fragments with ligated adaptors on both ends were enriched by PCR. PCR products were further purified using the AMPure XP system (Beckman Coulter, Beverly, USA) and quantified using the Agilent high sensitivity DNA assay on the Agilent Bioanalyzer 2100 system. Captured DNA fragments were sequenced on an Illumina NovaSeq 6000 platform (Illumina, San Diego, California).

Raw sequence files were assessed, trimmed, and mapped to the human genome reference sequence (UCSC hg19) using Novoalign V3.08.02 (Novocraft, Selangor, Malaysia). Variants were called jointly by GATK $3.8^{57}$ and annotation was performed using EPACTS (http://genome.sph.umich.edu/wiki/EPACTS) and Annovar ${ }^{58}$. The nomenclature of all variants studied was validated by using VariantValidator ${ }^{59}$

All single nucleotide variants were further filtered to obtain only high-quality variants. Briefly, quality control was carried out using the following parameters: (1) remove individual calls if Depth (DP) $<8$ or GenotypeQuality $(\mathrm{GQ})<20,(2)$ exclude variants if the average GQ value $\leq 35$, (3) exclude variants if call-rate value $\leq 0.9$, (4) keep only variants with no deviation from Hardy-Weinberg equilibrium ( $p>0.05$ after Bonferroni correction), (5) keep variants passing GATK VQSR (VQSRTranche of 90.0), (6) final hard filtering step with Quality by Depth $(\mathrm{QD}) \geq 2$, FisherStrand $(\mathrm{FS}) \leq 60$, RMSMappingQuality $(\mathrm{MQ}) \geq 40$,

MappingQualityRankSumTest (MQRankSum) $\geq-12.5$, ReadPosRankSumTest (ReadPosRankSum) $\geq-8$, StrandOddsRatio $(\mathrm{SOR}) \leq 3$ and ExcessHet $\leq 20$.

Association study on rare and low-frequency variants in RetNet genes. An association test was performed on rare and low-frequency non-synonymous variants from a curated RetNet list $(N=228$ genes, Suppl. Table 2$)$ to test possible association of variants in the 28 patients carrying rs118031911/T heterozygously compared to 3554 controls from the ToMMo database ${ }^{31}$. More specifically, variants were retained if they had a frequency comprised between 0.1 and $5 \%$ in controls, with the exclusion of rs118031911/T itself, according to published methods ${ }^{60-62}$, and in particular to the paper by Marouli and coworkers ${ }^{60}$. This resulted in the selection of 178 variants in 84 different genes, which were used to test association in rs118031911/T carriers vs. controls by Fisher's exact test with an experimentwide Bonferroni-corrected threshold of $2.81 \times 10^{-4}$, for $\alpha=0.05(0.05 / 178=$ $\left.2.81 \times 10^{-4}\right)$. $P$-values and odds ratios were obtained by the fisher.test function with default parameters in R (v3.5.1) and the Q-Q plot (Fig. 2) was obtained by using the qqman package ${ }^{63}$.

Relatedness analyses. PLINK (v1.90b5) ${ }^{64}$ was used to compute PI_HAT values between all pairs of the $28 \mathrm{rs} 118031911 / \mathrm{T}$ carriers using calls from whole exome sequencing. This analysis showed no relatedness, with PI_HAT values between 0.00 and 0.07 (threshold for relatedness $=0.2)^{65}$, for all 378 possible pairwise combinations (Supplementary Data 1 )

SNP genotyping of the RP1 locus. To detect haplotypes in trans with respect to rs118031911/T, we genotyped 23 SNPs encompassing the $R P 1$ locus over $\sim 260 \mathrm{~kb}$, by standard techniques.

\section{Data availability}

The data supporting the findings of this study, as a whole, contain information that could compromise the privacy/consent of the participants, therefore we provide the genotypes of the cases analyzed as a summary statistics file containing aggregated data (Supplementary Data 2). Genotypes of control Japanese individuals from the ToMMo Japanese Reference Panel Project (3.5KJPN release, v20181105open) can be accessed at https://jmorp.megabank.tohoku.ac.jp/201905/downloads. Data from the BioBank Japan Project and the Tohoku University School of Medicine and Yokohama City University Graduate School of Medicine is available upon request to the corresponding author, pending authorization of the Centers that generated them and in agreement with their specific IRB approvals.

\section{Code availability}

Processing of raw sequence files, variant calling, variant annotation, as well as all the other analyses described in this work were performed using available and open source software, as detailed in the Methods section. No custom code was therefore used, aside from simple scripts integrating commands for such programs.

Received: 21 September 2018 Accepted: 23 May 2019

Published online: 28 June 2019

\section{References}

1. den Hollander, A. I., Black, A., Bennett, J. \& Cremers, F. P. Lighting a candle in the dark: advances in genetics and gene therapy of recessive retinal dystrophies. J. Clin. Invest. 120, 3042-3053 (2010).
2. Berger, W., Kloeckener-Gruissem, B. \& Neidhardt, J. The molecular basis of human retinal and vitreoretinal diseases. Prog. Retin. Eye Res. 29, 335-375 (2010).

3. Runhart, E. H. et al. The common ABCA4 Variant p.Asn1868Ile shows nonpenetrance and variable expression of stargardt disease when present in trans with severe variants. Invest. Ophthalmol. Vis. Sci. 59, 3220-3231 (2018)

4. Zernant, J. et al. Frequent hypomorphic alleles account for a significant fraction of ABCA4 disease and distinguish it from age-related macular degeneration. J. Med. Genet. 54, 404-412 (2017).

5. Jin, Z. B. et al. Identifying pathogenic genetic background of simplex or multiplex retinitis pigmentosa patients: a large scale mutation screening study. J. Med. Genet. 45, 465-472 (2008).

6. Khan, M. I. et al. The molecular basis of retinal dystrophies in pakistan. Genes 5, 176-195 (2014)

7. Yamaguchi-Kabata, Y. et al. Japanese population structure, based on SNP genotypes from 7003 individuals compared to other ethnic groups: effects on population-based association studies. Am. J. Hum. Genet. 83, 445-456 (2008).

8. Nishiguchi, K. M. \& Rivolta, C. Genes associated with retinitis pigmentosa and allied diseases are frequently mutated in the general population. PLoS One 7, e41902 (2012).

9. Rivolta, C., Sharon, D., DeAngelis, M. M. \& Dryja, T. P. Retinitis pigmentosa and allied diseases: numerous diseases, genes, and inheritance patterns. Hum. Mol. Genet. 11, 1219-1227 (2002).

10. den Hollander, A. I. \& de Jong, E. K. Highly penetrant alleles in age-related macular degeneration. Cold Spring Harb. Perspect. Med. 5, a017202 (2014).

11. Katsanis, N. et al. Triallelic inheritance in Bardet-Biedl syndrome, a Mendelian recessive disorder. Science 293, 2256-2259 (2001).

12. Katsanis, N. The continuum of causality in human genetic disorders. Genome Biol. 17, 233 (2016).

13. Badano, J. L. \& Katsanis, N. Beyond Mendel: an evolving view of human genetic disease transmission. Nat. Rev. Genet. 3, 779-789 (2002).

14. Pierce, E. A. et al. Mutations in a gene encoding a new oxygen-regulated photoreceptor protein cause dominant retinitis pigmentosa. Nat. Genet. 22, 248-254 (1999).

15. Guillonneau, X. et al. A nonsense mutation in a novel gene is associated with retinitis pigmentosa in a family linked to the RP1 locus. Hum. Mol. Genet. 8, 1541-1546 (1999).

16. Sullivan, L. S. et al. Mutations in a novel retina-specific gene cause autosomal dominant retinitis pigmentosa. Nat. Genet. 22, 255-259 (1999).

17. Khaliq, S. et al. Novel association of RP1 gene mutations with autosomal recessive retinitis pigmentosa. J. Med. Genet. 42, 436-438 (2005).

18. Avila-Fernandez, A. et al. Identification of an RP1 prevalent founder mutation and related phenotype in Spanish patients with early-onset autosomal recessive retinitis. Ophthalmology 119, 2616-2621 (2012).

19. El Shamieh, S. et al. Targeted next generation sequencing identifies novel mutations in RP1 as a relatively common cause of autosomal recessive rodcone dystrophy. Biomed. Res. Int. 2015, 485624 (2015).

20. Baum, L. et al. RP1 in Chinese: Eight novel variants and evidence that truncation of the extreme $\mathrm{C}$-terminal does not cause retinitis pigmentosa. Hum. Mutat. 17, 436 (2001).

21. Ziviello, C. et al. Molecular genetics of autosomal dominant retinitis pigmentosa (ADRP): a comprehensive study of 43 Italian families. J. Med. Genet. 42, e47 (2005).

22. Liu, Q. et al. Identification and subcellular localization of the RP1 protein in human and mouse photoreceptors. Invest. Ophthalmol. Vis. Sci. 43, 22-32 (2002).

23. Liu, Q., Zuo, J. \& Pierce, E. A. The retinitis pigmentosa 1 protein is a photoreceptor microtubule-associated protein. J. Neurosci. 24, 6427-6436 (2004).

24. Iwanami, M., Oshikawa, M., Nishida, T., Nakadomari, S. \& Kato, S. High prevalence of mutations in the EYS gene in Japanese patients with autosomal recessive retinitis pigmentosa. Invest. Ophthalmol. Vis. Sci. 53, 1033-1040 (2012).

25. Suto, K. et al. Clinical phenotype in ten unrelated Japanese patients with mutations in the EYS gene. Ophthalmic Genet. 35, 25-34 (2014).

26. Arai, Y. et al. Retinitis Pigmentosa with EYS mutations is the most prevalent inherited retinal dystrophy in Japanese Populations. J. Ophthalmol. 2015, 819760 (2015).

27. Lek, M. et al. Analysis of protein-coding genetic variation in 60,706 humans Nature 536, 285-291 (2016).

28. Hildebrandt, F., Benzing, T. \& Katsanis, N. Ciliopathies. N. Engl. J. Med. 364, $1533-1543$ (2011).

29. Badano, J. L. et al. Dissection of epistasis in oligogenic Bardet-Biedl syndrome. Nature 439, 326-330 (2006)

30. Lindstrand, A. et al. Copy-number variation contributes to the mutational load of Bardet-Biedl syndrome. Am. J. Hum. Genet. 99, 318-336 (2016).

31. Nagasaki, M. et al. Rare variant discovery by deep whole-genome sequencing of 1,070 Japanese individuals. Nat. Commun. 6, 8018 (2015) 
32. Hanany, M. \& Sharon, D. Allele frequency analysis of variants reported to cause autosomal dominant inherited retinal diseases question the involvement of $19 \%$ of genes and $10 \%$ of reported pathogenic variants. J. Med. Genet. (2019).

33. Ji, W. et al. Rare independent mutations in renal salt handling genes contribute to blood pressure variation. Nat. Genet. 40, 592-599 (2008).

34. Sykiotis, G. P. et al. Oligogenic basis of isolated gonadotropin-releasing hormone deficiency. Proc. Natl Acad. Sci. USA 107, 15140-15144 (2010).

35. Kousi, M. et al. Evidence for secondary-variant genetic burden and nonrandom distribution across biological modules in a recessive ciliopathy. Preprint at https://www.biorxiv.org/content/10.1101/362707v1.full (2018).

36. Momozawa, Y. et al. Resequencing of positional candidates identifies low frequency IL23R coding variants protecting against inflammatory bowel disease. Nat. Genet. 43, 43-47 (2011).

37. Klassen, T. et al. Exome sequencing of ion channel genes reveals complex profiles confounding personal risk assessment in epilepsy. Cell 145, 1036-1048 (2011).

38. Stessman, H. A. et al. Targeted sequencing identifies 91 neurodevelopmentaldisorder risk genes with autism and developmental-disability biases. Nat. Genet. 49, 515-526 (2017).

39. Ebermann, I. et al. PDZD7 is a modifier of retinal disease and a contributor to digenic Usher syndrome. J. Clin. Invest. 120, 1812-1823 (2010).

40. Kajiwara, K., Berson, E. L. \& Dryja, T. P. Digenic retinitis pigmentosa due to mutations at the unlinked peripherin/RDS and ROM1 loci. Science 264, 1604-1608 (1994).

41. Yoshimura, $\mathrm{H}$. et al. Massively parallel DNA sequencing facilitates diagnosis of patients with Usher syndrome type 1. PLoS ONE 9, e90688 (2014).

42. Beales, P. L. et al. Genetic interaction of BBS1 mutations with alleles at other BBS loci can result in non-Mendelian Bardet-Biedl syndrome. Am. J. Hum. Genet. 72, 1187-1199 (2003).

43. Zaghloul, N. A. \& Katsanis, N. Functional modules, mutational load and human genetic disease. Trends Genet. 26, 168-176 (2010).

44. Tory, K. et al. High NPHP1 and NPHP6 mutation rate in patients with Joubert syndrome and nephronophthisis: potential epistatic effect of NPHP6 and AHI1 mutations in patients with NPHP1 mutations. J. Am. Soc. Nephrol. 18, 1566-1575 (2007).

45. Leitch, C. C. et al. Hypomorphic mutations in syndromic encephalocele genes are associated with Bardet-Biedl syndrome. Nat. Genet. 40, 443-448 (2008).

46. Louie, C. M. et al. AHI1 is required for photoreceptor outer segment development and is a modifier for retinal degeneration in nephronophthisis. Nat. Genet. 42, 175-180 (2010).

47. Alfano, G. et al. EYS is a protein associated with the ciliary axoneme in rods and cones. PLoS ONE 11, e0166397 (2016).

48. $\mathrm{Lu}, \mathrm{Z}$. et al. Ablation of EYS in zebrafish causes mislocalisation of outer segment proteins, F-actin disruption and cone-rod dystrophy. Sci. Rep. 7, 46098 (2017)

49. Messchaert, M. et al. Eyes shut homolog is important for the maintenance of photoreceptor morphology and visual function in zebrafish. PLoS ONE 13, e0200789 (2018).

50. Goldberg, A. F., Moritz, O. L. \& Williams, D. S. Molecular basis for photoreceptor outer segment architecture. Prog. Retin. Eye Res. 55, 52-81 (2016).

51. Liu, Q., Lyubarsky, A., Skalet, J. H., Pugh, E. N. Jr. \& Pierce, E. A. RP1 is required for the correct stacking of outer segment discs. Invest. Ophthalmol. Vis. Sci. 44, 4171-4183 (2003).

52. Genomics_England. The 100,000 Genomes Project Protocol v3. (2017).

53. Priest, J. R. et al. De novo and rare variants at multiple loci support the oligogenic origins of atrioventricular septal heart defects. PLoS Genet. 12, e1005963 (2016).

54. Katsanis, N. The oligogenic properties of Bardet-Biedl syndrome. Hum. Mol. Genet. 13, R65-R71 (2004).

55. Drmanac, R. et al. Human genome sequencing using unchained base reads on self-assembling DNA nanoarrays. Science 327, 78-81 (2010).

56. Nishiguchi, K. M. et al. Whole genome sequencing in patients with retinitis pigmentosa reveals pathogenic DNA structural changes and NEK2 as a new disease gene. Proc. Natl Acad. Sci. USA 110, 16139-16144 (2013).

57. McKenna, A. et al. The genome analysis toolkit: a MapReduce framework for analyzing next-generation DNA sequencing data. Genome Res. 20, 1297-1303 (2010).

58. Wang, K., Li, M. \& Hakonarson, H. ANNOVAR: functional annotation of genetic variants from high-throughput sequencing data. Nucleic Acids Res. 38, el64 (2010).

59. Freeman, P. J., Hart, R. K., Gretton, L. J., Brookes, A. J. \& Dalgleish, R. VariantValidator: accurate validation, mapping, and formatting of sequence variation descriptions. Hum. Mutat. 39, 61-68 (2018).

60. Marouli, E. et al. Rare and low-frequency coding variants alter human adult height. Nature 542, 186-190 (2017).
61. Chang, J. et al. Exome-wide analyses identify low-frequency variant in CYP26B1 and additional coding variants associated with esophageal squamous cell carcinoma. Nat. Genet. 50, 338-343 (2018).

62. Do, R. et al. Exome sequencing identifies rare LDLR and APOA5 alleles conferring risk for myocardial infarction. Nature 518, 102-106 (2015).

63. Turner, S. D. qqman: an R package for visualizing GWAS results using Q-Q and manhattan plots. Preprint at https://www.biorxiv.org/content/early/2014/ 05/14/005165 (2014).

64. Purcell, S. et al. PLINK: a tool set for whole-genome association and population-based linkage analyses. Am. J. Hum. Genet. 81, 559-575 (2007).

65. Grove, J. et al. Identification of common genetic risk variants for autism spectrum disorder. Nat. Genet. 51, 431-444 (2019).

\section{Acknowledgements}

This work was supported by the following agencies. The Swiss National Science Foundation (grant \#176097, to C.R.); the Rotterdamse Stichting Blindenbelangen, the Stichting voor Ooglijders and the Nelly Reef fund (to M.I.K. and F.P.M.C.); the Japan Agency for Medical Research and Development (grant \#17ek0109213h0001) and the Japan Society for the Promotion of Science (grant \#16K11315, to K.M.N.); Research on Measures for Intractable Diseases, Comprehensive Research on Disability Health and Welfare, the Strategic Research Program for Brain Science, and the Initiative on Rare and Undiagnosed Diseases (all to N.Ma.); the Japanese Agency for Medical Research and Development, Grants-in-Aid for Scientific Research (to N.Ma. and N.Mi.); the BioBank Japan Project, by the Japanese Ministry of Education, Culture, Sport, Science and Technology and the Japanese Agency for Medical Research and Development (to M.K.); the PhD Fellowship in Life Sciences (Faculty of Biology and Medicine, University of Lausanne), to M.Q. Most HRD patients were recruited from the Japanese Retinitis Pigmentosa Registry Project (JRPRP). The authors would also like to warmly thank Dr. Matthew Robinson for his expert advice on this work.

\section{Author contributions}

K.N., K.C., M.Q., and C.R. designed the research, wrote the manuscript, analyzed all the data, and interpreted the results. H.K.K. performed the analyses of the WGS sample and segregation analyses of the families. N.Mi. screened control individuals for the RP1 mutations. P.F., A.U.R., and K.N. performed Sanger sequencing analyses. A.P. contributed to the statistical analyses, while M.A., F.M., and K.M.N. analyzed the data. Under the coordination of K.M.N. and T.N., Y.I., S.U., N.F., A.M., Y.W., H.T., K.-H.S., T. I., K.M.N., and T.N. examined the patients and collected biological samples for genetic analysis. Y.K., C.T., N.Ma., and M.K. provided access to databases of control individuals M.I.K. and F.P.M.C. provided part of the sequencing data. Z.K. supervised the statistical analysis and wrote part of the manuscript. C.R. was the principal investigator of this study. All authors read and commented on the article.

\section{Additional information}

Supplementary Information accompanies this paper at https://doi.org/10.1038/s41467019-10746-4.

\section{Competing interests: The authors declare no competing interests.}

Reprints and permission information is available online at http://npg.nature.com/ reprintsandpermissions/

Peer review information: Nature Communications thanks the anonymous reviewers for their contribution to the peer review of this work.

Publisher's note: Springer Nature remains neutral with regard to jurisdictional claims in published maps and institutional affiliations.

Open Access This article is licensed under a Creative Commons Attribution 4.0 International License, which permits use, sharing, adaptation, distribution and reproduction in any medium or format, as long as you give appropriate credit to the original author(s) and the source, provide a link to the Creative Commons license, and indicate if changes were made. The images or other third party material in this article are included in the article's Creative Commons license, unless indicated otherwise in a credit line to the material. If material is not included in the article's Creative Commons license and your intended use is not permitted by statutory regulation or exceeds the permitted use, you will need to obtain permission directly from the copyright holder. To view a copy of this license, visit http://creativecommons.org/ licenses/by/4.0/

(C) The Author(s) 2019 